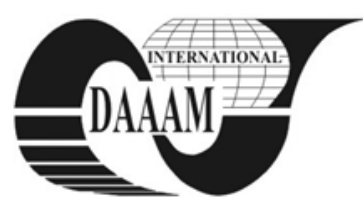

\title{
RESEARCH INTO THE UTILIZATION OF AN INERTIAL NAVIGATION SYSTEM IN ROBOTICS
}

\author{
BOZEK, P[avol] \& SURIANSKY, J[ozef]
}

\begin{abstract}
The paper deals with constructing the inertial navigation system (hereafter INS) which will be utilized for the calibration of a robotic workplace. The calibration is necessary for adapting the simulation of a production device model to real geometric conditions. The goal is to verify experimentally the proposed inertial navigation system in real conditions of the industrial robot operation.
\end{abstract}

Keywords: inertial system, micromechanical sensor, calibration, industrial robot

\section{INTRODUCTION}

The integration of navigational information represents the actual issue of reaching higher accuracy of required navigational parameters by using more, less accurate navigation systems. The inertial navigation is the navigation based on uninterrupted evaluating of the position of a navigated object with utilizing the sensors which are sensitive to motion, i.e. gyroscopes and accelerometers, which are regarded as primary inertial sensors or other sensors located on the navigated object. The position, orientation, direction and velocity of motion without external sources of information about the motion are constantly determined by means of the navigation computer and data from sensors. The actual position of the object is evaluated on the basis of knowledge of the initial position and subsequent continual measuring the acceleration and direction of motion in a reference system.. The principle of inertial navigation obeys the laws of classical mechanics defined by Newton. The INS includes at least one navigation computer and a platform or a module containing accelerometers and gyroscopes. From the constructional point of view inertial navigation systems are divided into platform so-called gimballed systems and nonplatform so-called strapdown systems. In the platform system inertial sensors are attached to the platform which is installed in a gimbals suspension with three degrees of freedom with the aim of remaining the constant space orientation in defined directions (north - south, east - west and vertically on performing the gravitational attraction), while the gimbals suspension is firmly connected to the construction of the navigated object. The moving mechanical parts of the systems cause relatively low reliability towards the non-platform systems. The inertial sensors of non-platform systems are firmly connected to the construction of the object (usually in the centre), for whose navigation they are determined. Both types of the inertial navigation systems consist of an inertial measurement unit and a navigation computer.

The aim of the research is to investigate and develop a new combined inertial navigation system based on electronic gyroscopes, magnetic and barometric sensors. The mentioned system will ensure the accuracy which is necessary for the calibration of robotic workplaces and thereby the necessities of utilizing the calibration agents will be limited. A big advantage of the INS is also its autonomy in comparison with methods used nowadays. This leads to the essential simplification of calibration and it even carries big possibilities with it in the field of control and measuring, for example, avoiding the accidental collisions of robots etc. To solve a problem of ensuring the required accuracy is a basic problem. The integration of more measuring devices (INS) is one of the possibilities (Sotak et al., 2006). The integration of navigation information represents the topical issue of achieving greater accuracy of required navigation parameters. The crucial activity is focused on three basic fields:

- The first goal is to analyze accelerometer and gyroscopic sensors and their possibilities of utilization for inertial navigation. The simulation of the effect of sensors with different metrological parameters and their effect on the properties of the proposed combined navigation system.

- The second goal is to optimize a specialized processor system for processing the data from the defined sensors in connection with controlling items of an industrial robot (Benes et al., 2009). The proposal of an algorithm of combined navigation with respect to the used processor system.

- The third goal is to verify experimentally the proposed inertial navigation system in real conditions of the industrial robot operation.

\section{CHARACTERISTIC OF THE ISSUE}

The demand of navigation autonomy, i.e. the independence of the external sources of navigation information became the reason for implementing the inertial navigation systems. The principle of inertial navigation is based on Newton's laws which express a change of motion under the action of external forces and acceleration which is directionally and by size proportional to the acting external force. The inertial navigation system consists of a measurement unit containing accelerometers and gyroscopes and from a navigation computer which evaluates the data from measuring devices. In contrast to all the other navigation systems inertial navigation is completely autonomous, self-sustaining and independent of the surrounding environment, i.e. the system is resistant to outside influences such as magnetic disturbances, electronic interference and signal distortion. Computing operations in the inertial navigation system are based on Newton's law of motion.

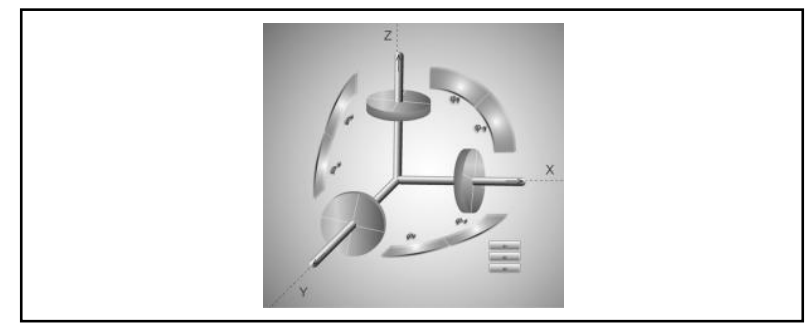

Fig. 1. Basic principle of INS activity

For the purpose of navigation in a coordinate system it is necessary to keep the direction of motion in the direction of acceleration. This is not practically possible, and therefore sensors - gyroscopes are used for detecting the rotary motion. 
Seeing that each free object in space has six degrees of freedom (internally mutually independent variables) the inertial navigation system usually consists of three gyroscopes and three accelerometers where each pair (gyroscope, accelerometer) is able to record the rotation or acceleration in the direction of one axis which is perpendicular to the others. Of the six degrees it is three linear degrees of freedom (Fig. 1), the translation in the $\mathrm{X}$-axis, $\mathrm{Y}$-axis and $\mathrm{Z}$-axis which indicate the position of the object and three degrees of freedom of rotation which indicate rotating around the $\mathrm{X}$-axis, $\mathrm{Y}$-axis, and Z-axis.

The position of the object is also known if we know the six variables. If this data is observed for a certain period of time, it is possible to determine the trajectory and speed of an object's motion from it. The electronic gyroscope is one of the most modern gyroscopic sensors. The mentioned sensors use the Coriolis force which acts on the particle moving with certain speed in a rotating non-inertial reference system and which is directly proportional to the absolute value of the angular velocity vector of this system (Stollmann, 2007). The Coriolis force acts on the resonating mass, which is flexibly embedded in the frame and when the frame turns, in the direction perpendicular to the axis of rotation (perpendicularly to the plane of the frame) and perpendicular to the direction of motion of resonating mass. The Coriolis force also alternates its orientation in the direction perpendicular to the direction of motion because the resonating mass oscillates in one direction. The amplitude of this force is measured by means of a change of electric capacity of a condenser whose electrodes are connected to the stable and movable frame.

The inertial measurement unit (IMU) is an essential item of each INS. Sensors, whose output is influenced by the motion of the object on which the IMU is placed, are regarded as primary sensors of the IMU. Primary sensors in inertial navigation are sensors of angular velocity, whose output signals after integration are used for determining the orientation in space, and accelerometers whose output signals after precise compensation of gravitational acceleration and the Coriolis force can be integrated onto the speed and position. Such an inertial measurement unit has six degrees of freedom. This means it enables to measure translational and rotary motion in three orthogonal axes. The accuracy of inertial sensors plays a key role in autonomous navigation. Errors of current inertial sensors have the approximate value of $0.01^{\circ}$ hour for gyroscopes and $100 \mu \mathrm{g}$ for accelerometers (King, 2009). The mentioned errors are integrated in time and cause the error of determining the position which is expressed by the nonaccuracy of measuring per hour, which is, however, minimal. Such high-power IMU are implemented only into the inertial navigation systems for special use.

The mutual integration of accelerometers, gyroscopes, magnetometers, barometric sensors and microprocessor items into a compact unit, whose output values is the data about position, rotation, height and the like, is a current trend in the development and production of inertial units. Basic inertial sensors are supplemented with a GPS module or magnetic sensor to compensate the errors of inertial sensors.

\section{BENEFITS OF SOLUTION}

In contemporary space requirements and the effective utilization of space the robot often has to work in confined conditions. Moving a tool or manipulating with parts requires great accuracy. The enormous demands for the correct calibration of a robot device result from this. The calibration will fundamentally be simplified by using the INS in the field of calibration. The contribution in the field of control and measurement, for example, preventing the accidental collisions of robots, will be significant.
It is possible to expect the next contribution in the original proposal of the algorithm of combined navigation with respect to the used processor system and its optimization in connection to the use in robotics and connection to the operation of robots mainly on their safety and economics. Description of used methodological procedures - modern methods, analyses and simulations with the use of modern software tools such as OrCAD, Matlab, Multisim will be used in mentioned fields (Zolotova et al., 2005). The shape of stationary magnetic fields will be investigated on an experimental laboratory model which uses an area positioning system. Possibilities of using the magnetoresistance sensors and barometric sensors will be verified in the sensor system (Cupec et al., 2009). The original own algorithms will be utilized in the course of processing the data from the sensors. The solution implements planned experimental verification of the navigation system first on a model robot, and later, in real operation conditions of a robot device with evaluating the uncertainties.

The output is the original construction of the navigation system utilizable in the operation of robots - the elaboration of the study with the possibility of implementation of inertial navigation for the calibration of robotic workplaces, the system of control with a possibility of avoiding collisions during the operation.

\section{CONCLUSION}

The possibilities of utilizing the inertial systems are directly proportional to the advance in their development. The ability of precise measuring the position of the robot mainly in necessarily regularly repetitive calibration is increased by this. The implemented INS is able to measure accelerating and slewing the watched point of the arm and to use it for determining the position of the robotic arm in space (Hromada et al., 1997).

\section{ACKNOWLEDGEMENTS}

The contribution was elaborated within the research project KEGA project No. 3-7285-09 Contents Integration and Design of University Textbook "Specialised Robotic Systems" in Print and Interactive Modules for University of Technology in Zvolen, Trenčín University and Slovak University of Technology in Bratislava.

\section{REFERENCES}

Benes, L.; Kaloc, R. \& Minar, L. (2009), Contact deformation generation and properties of mechanically loaded surfaces. Machine Manufacturing, 2009, 68-75. ISSN 0016-8580

Cupec, R.; Denk, J. \& Schmidt, G. (2002). Practical Experience with Vision-Based Biped Walking, Proceedings of the 8th International Symposium on Experimental Robotics, (ISER), Italija, log. no. 083

Hromada, J.; Bubenik, P. \& Hradnansky, M. (1997). Simulation as a supporting tool for the election management system. 19th International Workshop. Ostrava: MARQ, pp.283-288. ISBN 80-85988-20-8

King, A. (2009). Inertial Navigation / Forty Years of Evolution

Sotak, M. et al. (2006). Integration of navigation systems. 1. ed. Košice

Stollmann,V. (2007). Robotics technology sharing process. In: Information technology and automation in process control, III. Scientific Conference, 12.6.2007, TU Zvolen, ISBN 978-80-228-1759-2

Zolotova, I.; Flochova, J. \& Ocelikova, E. (2005). Database Technology and Real Time Industrial Transaction Techniques in Control. Journal of Cybernetics and Informatics, Volume 5, Slovakia, Bratislava, ISSN 13364774. pp. 18-23 http://www.sski.sk/yournal/index.php 